\title{
A Survey on Multi-label Classification for Images
}

\author{
Radhika Devkar \\ Department of Computer Engineering \\ Marathwada Mitra Mandal's College of \\ Engineering, Pune.
}

\author{
Sankirti Shiravale \\ Department of Computer Engineering \\ Marathwada Mitra Mandal's College of \\ Engineering, Pune.
}

\begin{abstract}
The area of an image multi-label classification is increase continuously in last few years, in machine learning and computer vision. Multi-label classification has attracted significant attention from researchers and has been applied to an image annotation. In multi-label classification, each instance is assigned to multiple classes; it is a common problem in data analysis. In this paper, represent general survey on the research work is going on in the field of multilabel classification. Finally, paper is concluded towards challenges in multi-label classification for images for future research.
\end{abstract}

\section{General Terms}

Machine Learning, Image Classification

\section{Keywords}

Multi-label Classification, Image annotation, machine learning, computer vision

\section{INTRODUCTION}

Multi-label classification for images is a task of great significance in the field of computer vision and machine learning. Nowadays, great amount of digital images present in the world. Today images are important way for information gaining, appearing and transmitting because of its visual foundation for recognizing the world. Image classification provides great support for image retrieval and also indexing because both of them require accurately label images. There are two ways for labeled classifications of images are single label classification and multi label classification. In single label image classification, each image have single class label, but there are multiple label class are available. In multi-label image classification, each image has multiple class labels at the same time. One of the ways of image classification is binary image classification where image is classified between one of the two mentioned classes. But sometimes images contain various target labels; this will increase the complexity of implementation of the multi-label classification as to identify labels for images. Based on the label association to the input samples, the classification methods can be categorized into single-label classification and multi-label classification [6], [7]. Single label classification is an approximate function that associates the input samples to a unique target label 1 from a set of disjoint labels L. The singlelabel classification problem can be further divided into two categories: Binary and multi-class classification. When the input data samples are categorized into one of two classes, it is called binary classification. When the input samples correspond to one among a pool of target labels, it is called multi-class classification. Binary classification is the most basic classification and forms the basic requirement a technique should fulfill to be a classification method. Hence, all the classification methods and the variants available in the literature thus far can be used for binary classification. Use single-label classification framework for label the above image. Refer fig. 1, using single label classification user can label it as sea. But there are multiple objects present in it like, tree, sky, etc. but in single label classification only single label is for one image. User can search or retrieve this image using only one label i.e. sea.

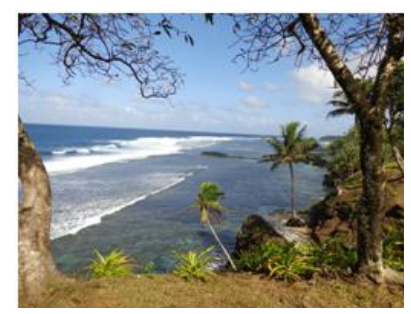

Fig.1. Example image

Multi-label classification has gained much importance in recent years due to its wide range of application domains. As opposed to single-label classification, each input sample is associated with a set of target labels in multi-label classification. The number of target labels corresponding to each input is not fixed and varies dynamically. This results in increased complexity in the implementation of multi-label classifier. Use multi-label classification framework for label the above image. Refer fig. 1, using multi-label classification user can label it as set of labels like, sea, tree, sea, etc. user can label single image with multiple labels. If user wants to search or retrieve this he or she can use one of the above mentioned labels.

In this paper, first section will be review of literature where discuss some multi-label classification method and related work, and then in second section explore some challenges in multi-label classification and at the end conclusion with future work.

\section{REVIEW OF LITERATURE}

Research on multi-label learning originates from text categorization. Recent decades have witnessed great progress in multi-label classification algorithms. Nowadays, multilabel classification algorithms [5] are used in image classification. The focus of this work is more on labels of images. Multiple labels are usually associated with an image in many image analysis applications and there are several kinds of methods for addressing multi-label problem.

Machine learning approaches are used to assign semantic labels to images automatically through a supervised classifier. In supervised classifier, the input measure can be mapped to the known class labels. Supervised learning is to predict the value of an outcome measure based on a set of input measure. Two phases are included: training and testing phase. During the training phase, through the supervised model, the training sets are classified into classes. During the testing phase, unknown instance are mapped into one of the class labels in the training set. The common machine learning techniques 
under supervised learning are support vector machines, artificial neural networks and decision tree.

\subsection{Multi-label Classification Techniques \\ 2.1.1 ML-kNN}

ML-Knn [7] is derived from the popular k-Nearest Neighbor $(\mathrm{kNN})$ algorithm. First, for each test instance, its $\mathrm{k}$ nearest neighbors in the training set is identified. Then, according to statistical information gained from the label sets of these neighboring instances, i.e. the number of neighboring instances belonging to each possible class, maximum a posteriori (MAP) principle is utilized to determine the label set for the test instance.

\subsubsection{Multi-label Decision Tree}

The basic idea of this algorithm is to adopt decision tree [30] techniques to deal with multi-label data, where an information gain criterion based on multi-label entropy is utilized to build the decision tree recursively. Some algorithms of decision tree have developed such as ID3, C4.5 and CART. ID3 and C4.5 are only used for classification; where CART is used for classification and numerical prediction also.

\subsubsection{Ranking SVM}

The basic idea of this algorithm is to adapt maximum margin strategy to deal with multi-label data, where a set of linear classifiers are optimized to minimize the empirical ranking loss. Using hyper-plane, RankSVM[16],[21],[22],[23],[27] classify the label pair into relevant and irrelevant label pairs.

Table 1. Comparison between Multi-Label Classification Techniques

\begin{tabular}{|l|l|l|l|}
\hline $\begin{array}{c}\text { Sr. } \\
\text { No. }\end{array}$ & Methods & \multicolumn{1}{|c|}{ Pros } & \multicolumn{1}{|c|}{ Cons } \\
\hline 1 & ML-KNN & $\begin{array}{l}\text { Decision boundary } \\
\text { is adaptively } \\
\text { adjustable, Reduce } \\
\text { class imbalance } \\
\text { issues }\end{array}$ & $\begin{array}{l}\text { Exploration of } \\
\text { inter label } \\
\text { correlation is not } \\
\text { possible }\end{array}$ \\
\hline 2 & $\begin{array}{l}\text { Multi- } \\
\text { label } \\
\text { Decision } \\
\text { Tree }\end{array}$ & $\begin{array}{l}\text { Use semantic } \\
\text { rules, fast }\end{array}$ & Need pruning \\
\hline 3 & $\begin{array}{l}\text { Ranking } \\
\text { SVM }\end{array}$ & $\begin{array}{l}\text { Linear } \\
\text { classification, high } \\
\text { efficient }\end{array}$ & $\begin{array}{l}\text { Cannot find inter } \\
\text { label correlation, } \\
\text { Cannot handle } \\
\text { large label dataset }\end{array}$ \\
\hline
\end{tabular}

\subsection{Related Work}

Yunchao Wei et al. [2], propose Hypotheses-CNN-Pooling (HCP) for multi-label classification of images, where large single label image datasets are trained by CNN. Xuchun Li et al. [1], proposed a multi-label SVM active learning method and used it to solve multi-label image classification problem. Tao Zeng and Shuiwang Ji [3], resolve multi-task problem using multi-instance multi-task CNN. In CNN model, at image level transfer prior knowledge learned from large single label single task data sets. Xinmiao Ding et al.[18], proposed a context-aware multi-instance multi-label learning (CMIML) model consider instances and labels two cues. Using multiple graphs construct instance context cues and linearly combine labels are used to construct label context cues. These two context cues are integrated into a united framework.
Xin $\mathrm{Li}$ and Yuhong Guo[21], proposed multi-label classification strategy which is combination of label cardinality inconsistency and max-margin prediction uncertainty. Jian $\mathrm{Wu}$ et al. [20], represent selection technique for the most useful example-label pairs, but label correlation of an example is not consider. Jesse Read et al. [13], presented a single framework for multi-label classification using metalabels. Bo Wang et al.[19], proposed a dynamic label propagation (DLP), which improves multi-label classification using semi-supervised learning. Jiang Wang et al. [10], proposed a combine CNN-RNN framework for multi-label classification for images. Advantages of $\mathrm{CNN}$ and RNN are use for joint image or label co-occurrence embedding. Yanwei Pang et al. [17], proposed an image classification technique which latent topic information and use multi-label multiple kernel learning to improve the image classification. Benhui Chen et al.[16], proposed multi-label classification technique based on delicate decision boundary SVM which improves the label ranking. Teng $\mathrm{Li}$ et al.[15], for label ranking and image annotation application developed contextual image decomposition method. Yong Luo et al.[9], semi-supervised multi-label classification for image use manifold regularized multitask learning (MRMTL) based algorithm.

Xiangyang Xue et al.[14], proposed a framework in which multiple labels are obtain by using feature label association and inter label correlation. Co-occurrence matrix and structured max margin framework is used. Yan Huang et al. [12], multi-label learning transform into multiple binary classification tasks, and used deep neural network architecture to handle multiple task problem. Ricardo Cabral et al. [11], proposed a method in which, low rank problem is formulated using weakly supervised learning and use transductive matrix to solve this. Meixiang Xu et al. [8], to resolve multi-label learning problems proposed a method based on the supervised-regression with co-training style, efficiently exploit abundant unlabeled data. Aiwen Jiang et al.[22], proposed a calibrated RankSVM for multi-label classification. Using this technique multi-label classification and label ranking both problems are solved. Using RankSVM, a virtual label used as a calibrated scale, the rank algorithm has a natural zero-point. The optimal coefficient of the virtual label (threshold) is implanted in learning process, to obtain optimal position of the virtual labels adaptively. This makes difference between conventional RankSVM and calibrated Rank-SVM. Gulisong Nasierding et al.[23], discuss useful multi-label classification techniques for image and study clustering based multi-label classification (CBMLC) for the multi-label classification problem.

Xin $\mathrm{Li}$ et al.[24], represented model for multi-label image classification i.e. 'probabilistic label enhancement'. This model use the conditional random field framework, first construct a tree graph in the label space based on cooccurrence patterns of the labels in the training data, and then perform piecewise training. Gangadhara Rao Kommu et al.[29], proposed two probabilistic approaches partial and mutual information about labels to solve multi-label classification. Chen Ye et al. [25], proposed a method for multi-label active learning based on cosine similarity. Use cosine similarity to accurately evaluate the correlations between all labels. It reduces the labeling cost. But only positive correlation between all labels is considered not negative correlations. Jiwei $\mathrm{Hu}$ et al.[26], represented effective hierarchical image annotation system which use label propagation to the NBNN classifier. It effectively removes the irrelevant labels, and solves the multi-label problem. Wail Mustafa et al.[27], represented categorization 
technique for object to categorize into multiple and nested categories and it uses joint SVM to learn visual categories. Xiaoyu Zhang et al. [28], proposed a multi-label learning method, in which active learning and multi-label learning are combine together for multi-label image classification. Eva Gibaja et al. [30], presents a multi-label classification techniques based on decision tree by implementing J48 of C4.5.

In the following table 2, comparison of some of the methods used for multi-label classification is shown. The results are also provided in terms of accuracy, mAP and hamming loss of relevant label sets.

Table 2. Comparative study of Multi-label Classification

\begin{tabular}{|c|c|c|}
\hline Authors & Method Used & Results \\
\hline $\begin{array}{l}\text { Jiang Wang } \\
\text { et al.[10] }\end{array}$ & $\begin{array}{l}\text { Combine CNN and } \\
\text { RNN technique }\end{array}$ & mAP- $84.0 \%$ \\
\hline $\begin{array}{l}\text { Jian Wu et } \\
\text { al.[20] }\end{array}$ & $\begin{array}{l}\text { Example label based } \\
\text { multi-label active } \\
\text { learning method }\end{array}$ & Accuracy-8.7 \\
\hline $\begin{array}{l}\text { Yong Luo, } \\
\text { et al.[9] }\end{array}$ & $\begin{array}{l}\text { Use manifold } \\
\text { regularization for } \\
\text { multi-label task }\end{array}$ & mAP-0.410 \\
\hline $\begin{array}{l}\text { Xin Li et } \\
\text { al.[21] }\end{array}$ & $\begin{array}{l}\text { Use SVM classifier } \\
\text { with max margin } \\
\text { uncertainty and label } \\
\text { cardinality } \\
\text { inconsistency }\end{array}$ & Accuracy-0.33 \\
\hline $\begin{array}{l}\text { Benhui } \\
\text { Chen et } \\
\text { al.[16] }\end{array}$ & $\begin{array}{lr}\text { Use } & \text { probabilistic } \\
\text { SVM } & \text { classifier } \\
\text { delicate } & \text { decision } \\
\text { boundary } & \end{array}$ & $\begin{array}{l}\text { Accuracy- } \\
0.7263\end{array}$ \\
\hline $\begin{array}{l}\text { Yan Huang } \\
\text { et al.[12] }\end{array}$ & $\begin{array}{l}\text { Use deep neural } \\
\text { network for multiple } \\
\text { label task }\end{array}$ & $\begin{array}{l}\text { Hamming Loss- } \\
0.157 \pm 0.008\end{array}$ \\
\hline $\begin{array}{l}\text { Eva Gibaja } \\
\text { et al.[30] }\end{array}$ & $\begin{array}{l}\text { Use decision tree for } \\
\text { multi-label } \\
\text { classification }\end{array}$ & $\begin{array}{l}\text { Accuracy- } \\
0.595\end{array}$ \\
\hline
\end{tabular}

\section{CHALLENGES IN MULTI-LABEL CLASSIFICTION}

\subsection{Exploit the correlation between labels}

Main Challenge in multi-label classification is label dependency [4]. For each image there are multiple relevant labels are retrieve but finding dependency between labels is useful for machine learning. Label dependency helps to explore the correlation between two labels. There are many algorithms which help to explore the correlation between labels to increase the accuracy of the multi-label classifier; however complexity of the learning process of these algorithms is high. Based on that, the real challenge is to exploit high order labels correlations.

\subsection{In Multi-label Datasets, label space with high dimensionality}

High dimensionality [31] is one of the most challengeable issues in multi-label classification. Same situation is appeared in single label classification as a problem of imbalance class distribution. And the situation will be worse when the number of labels in the dataset is very high. There is need of fast and simple algorithm to handle large number of labels that are associated with some numbers of instances.

\section{CONCULTION}

In this paper, discussion of the brief introduction of multilabel classification for images. And we also discuss well known multi-label classification techniques. We survey some multi-label classification algorithms for images. With this, challenges in the multi-label classification are explained briefly. In the future, we aim to study in detail these challenges and propose new technique to exploit inter label correlation.

\section{REFERENCES}

[1] Xuchun Li, Lei Wang, Eric Sung, "Multi-Label SVM Active Learning for Image Classification", IEEE Image Processing ICIP '04, 2004.

[2] Yunchao Wei, Wei Xia, Min Lin, Junshi Huang, Bingbing Ni, Jian Dong, Yao Zhao, and Shuicheng Yan "HCP: A Flexible CNN Framework for Multi-label Image Classification", IEEE Transactions On Pattern Analysis And Machine Intelligence, 2015.

[3] Tao Zeng and Shuiwang Ji, "Deep Convolutional Neural Networks for Multi-Instance Multi-Task Learning", 2015 IEEE International Conference on Data Mining, Pages: 579-589, 2015.

[4] Sheng-Jun Huang and Zhi-Hua Zhou, "Multi-Label Learning by Exploiting Label Correlations Locally", Proceedings of the 26th AAAI Conference on Artificial Intelligence, Pages: 949-955, 2012.

[5] Min-Ling Zhang and Zhi-Hua Zhou, "A Review on Multi-Label Learning Algorithms", IEEE Transactions on Knowledge and Data Engineering, Volume: 26, No. 8, 2014.

[6] Meng Joo Er, Rajasekar Venkatesan, Ning Wang, "An Online Universal Classifier for Binary, Multi-class and Multi-label Classification" Cornell University Library, Pages: 1-6, 2016.

[7] G. Tsoumakas and I. Katakis, "Multi label Classification: An overview", International Journal of Data Warehousing and Mining, Volume: 3, No. 3, Pages: 113, 2007.

[8] Meixiang Xu, Fuming Sun, Xiaojun Jiang, "Multi-label learning with co-training based on semi-supervised regression", Proceedings 2014 IEEE International Conference on Security, Pattern Analysis, and Cybernetics (SPAC), Pages: 175 - 180, 2014.

[9] Yong Luo, Dacheng Tao, Bo Geng, Chao Xu, Stephen J. Maybank, "Manifold Regularized Multitask Learning for Semi-Supervised Multilabel Image Classification", IEEE Transactions on Image Processing, Volume: 22, Issue: 2, Pages: 523 - 536, 2013. 
[10] Jiang Wang, Yi Yang, Junhua Mao, Zhiheng Huang, Chang Huang, Wei $\mathrm{Xu}$, "CNN-RNN: A Unified Framework for Multi-label Image Classification", 2016 IEEE Conference on Computer Vision and Pattern Recognition (CVPR), Pages: 2285 - 2294, 2016.

[11] Ricardo Cabral, Fernando De la Torre, Joo Paulo Costeira, Alexandre Bernardino, "Matrix Completion for Weakly-Supervised Multi-Label Image Classification", IEEE Transactions on Pattern Analysis and Machine Intelligence, Volume: 37, Issue: 1, Pages: 121 - 135, 2015.

[12] Yan Huang, Wei Wang, Liang Wang, Tieniu Tan, "Multi-task deep neural network for multi-label learning”, 2013 IEEE International Conference on Image Processing, Pages: 2897 - 2900, 2013.

[13] Jesse Read, Antti Puurula, Albert Bifet, "Multi-label Classification with Meta-Labels", 2014 IEEE International Conference on Data Mining, Pages: 941 946, 2014.

[14] Xiangyang Xue, Wei Zhang, Jie Zhang, Bin Wu, Jianping Fan, Yao Lu, "Correlative multi- label multiinstance image annotation", 2011 International Conference on Computer Vision, Pages: 651 - 658, 2011.

[15] Teng Li, Shuicheng Yan, Tao Mei, Xian-Sheng Hua, InSo Kweon, "Image Decomposition With Multi-label Context: Algorithms and Applications", IEEE Transactions on Image Processing, Volume: 20, Issue: 8 , Pages: 2301 - 2314, 2011.

[16] Benhui Chen, Weifeng Gu, Jinglu $\mathrm{Hu}$, "An improved multi-label Classification based on label ranking and delicate boundary SVM", 2010 International Joint Conference on Neural Networks (IJCNN), Pages: 1 - 6, 2010.

[17] Yanwei Pang, Zhao Ma, Yuan Yuan, Xuelong Li, Kongqiao Wang, "Multimodal learning for multi-label image Classification", 2011 18th IEEE International Conference on Image Processing, Pages: 1797 - 1800, 2011.

[18] Xinmiao Ding, Bing Li, Weihua Xiong, Wen Guo, Weiming $\mathrm{Hu}$, Bo Wang, "Multi-Instance Multi-Label Learning Combining Hierarchical Context and its Application to Image Annotation", IEEE Transactions on Multimedia, Volume: 18, Issue: 8, Pages: 1616 - 1627, 2016.

[19] Bo Wang, Zhuowen Tu, John K. Tsotsos, "Dynamic Label Propagation for Semi-supervised Multi-class Multi-label Classification", 2013 IEEE International Conference on Computer Vision, Pages: 425 - 432, 2013.

[20] Jian Wu, Victor S. Sheng, Jing Zhang, Pengpeng Zhao, Zhiming Cui "Multi-label Active Learning for Image
Classification", 2014 IEEE International Conference on Image Processing (ICIP), Pages: 5227 - 5231, 2014.

[21] Xin Li and Yuhong Guo, "Active Learning with MultiLabel SVM Classification", IJCAI, Pages: 1479-1485, 2013.

[22] Aiwen Jiang, Chunheng Wang, Yuanping Zhu, "Calibrated Rank-SVM for Multi-Label Image Categorization”, IEEE, Pages: 1450-1456, 2008.

[23] Gulisong Nasierding and Atul Sajjanhar,"Multi-label Classification with Clustering for Image and Text Categorization", 2013 6th International Congress on Image and Signal Processing IEEE, Pages: 869-874, 2013.

[24] Xin Li, Feipeng Zhao and Yuhong Guo, "Multi-label Image Classification with A Probabilistic Label Enhancement Model", UAI'14, Proceedings of the Thirtieth Conference on Uncertainty in Artificial Intelligence, Pages 430-439, 2014.

[25] Chen Ye, Jian Wu, Victor S. Sheng, Pengpeng Zhao, and Zhiming Cui, "Multi-Label Active Learning with Label Correlation for Image Classification", ICIP 2015 IEEE, Pages: 3437-3441.

[26] Jiwei Hu, Kin Man Lam, Guoping Qiu, "A HIERARCHICAL ALGORITHM FOR IMAGE MULTI-LABELING”, IEEE 17th International Conference on Image Processing, Pages: 2349-2352, 2010.

[27] Wail Mustafa, Hanchen Xiong, Dirk Kraft, Sandor Szedmak, Justus Piater and Norbert Kruger, "MultiLabel Object Categorization Using Histograms of Global Relations", IEEE 2015 International Conference on 3D Vision, Pages: 309-317, 2015.

[28] Xiaoyu Zhang, Jian Cheng, Changsheng Xu, Hanqing $\mathrm{Lu}$, and Songde Ma, "MULTI-VIEW MULTI-LABEL ACTIVE LEARNING FOR IMAGE CLASSIFICATION", ICME 2009 IEEE, Pages: 258$261,2009$.

[29] Gangadhara Rao Kommu, M.Trupthi and Suresh Pabboju, "A Novel Approach for Multi-label Classification using Probabilistic Classifiers", IEEE International Conference on Advances in Engineering \& Technology Research, 2014

[30] Eva Gibaja, Manuel Victoriano, Jose Luis AvilaJimenez, and Sebastian Ventura, "A TDIDT Technique for Multi-label Classification”, IEEE 2010, 519-524, 2010.

[31] Raed Alazaidah and Farzana Kabir Ahmad, "Trending Challenges in Multi Label Classification", (IJACSA) International Journal of Advanced Computer Science and Applications, Vol. 7, Pages: 127-131, 2016. 